Abstract 69 Table 1 LIT values against T2T outcomes stratified by FM status in SLE

\begin{tabular}{|c|c|c|c|}
\hline Category & Estimated Mean LIT Score & standard error & $P$ value \\
\hline \multicolumn{4}{|c|}{ ALL $(n=1356)$} \\
\hline \multicolumn{4}{|l|}{ MODEL 1} \\
\hline Non Optimal & 34.57 & 0.98 & Ref \\
\hline LDAS/RONT & 28.22 & 0.65 & $<0.001$ \\
\hline ROFT & 24.61 & 1.1 & $<0.001$ \\
\hline \multicolumn{4}{|l|}{ MODEL 2} \\
\hline Non Optimal & 34.51 & 0.98 & Ref \\
\hline RONT & 30.43 & 0.94 & 0.0007 \\
\hline LDAS & 27.18 & 0.72 & $<0.001$ \\
\hline ROFT & 24.55 & 1.06 & $<0.001$ \\
\hline \multicolumn{4}{|c|}{ WITH FM $(n=76)$} \\
\hline \multicolumn{4}{|l|}{ MODEL 1} \\
\hline Non Optimal & 56.76 & 4.5 & ref \\
\hline LDAS/RONT & 48.43 & 2.7 & $<0.001$ \\
\hline ROFT & 35.95 & 4.4 & $<0.001$ \\
\hline \multicolumn{4}{|l|}{ MODEL 2} \\
\hline Non Optimal & 56.64 & 4.51 & ref \\
\hline RONT & 53.95 & 5.8 & 0.64 \\
\hline LDAS & 45.77 & 5.06 & 0.03 \\
\hline ROFT & 35.83 & 5.84 & 0.006 \\
\hline \multicolumn{4}{|c|}{ WITHOUT FM $(n=1280)$} \\
\hline \multicolumn{4}{|l|}{ MODEL 1} \\
\hline Non Optimal & 33.4 & 1 & ref \\
\hline LDAS/RONT & 27.1 & 0.7 & $<0.001$ \\
\hline ROFT & 23.8 & 1.1 & $<0.001$ \\
\hline \multicolumn{4}{|l|}{ MODEL 2} \\
\hline Non Optimal & 33.3 & 0.99 & Ref \\
\hline RONT & 29.1 & 1.22 & 0.0006 \\
\hline LDAS & 26.13 & 1.04 & $<0.0001$ \\
\hline ROFT & 23.76 & 1.37 & $<0.0001$ \\
\hline
\end{tabular}

LIT was able to differentiate between T2T outcomes, and the scores moved in the anticipated direction. Though LIT scores for RONT were lower than NO in those with FM, they did not reach significance. This could potentially be secondary to small number of FM patients and use of 4 comparator categories.

Conclusions LIT can differentiate between T2T outcomes in SLE patients with and without FM. Given its extensive validation in various languages, regions, measurement equivalence, ease of use, acceptability by patients and physicians, and responsiveness to changes in assessments by physician (SLEDAI, PGA, SRI, T2T) and patient (HRQOL, SLAQ), the tool is indicated for routine patient care, research and clinical trials.

Funding Source(s): None

\section{R848 (RESIQUIMOD), A TLR 7/8 AGONIST, ACCELERATES DISEASE AND CAUSES A FATAL MYELOPROLIFERATIVE DISORDER IN NZM 2410 LUPUS- PRONE MICE}

${ }^{1}$ Jena Wirth, ${ }^{2}$ Sheryl Coutermarsh-Ott, ${ }^{1}$ Ivan Molano, ${ }^{3}$ Gary S Gilkeson, ${ }^{4}$ Melissa A Cunningham*. ${ }^{1}$ MUSC; ${ }^{2}$ Department of Biomedical Sciences and Pathobiology, VirginiaMaryland College of Veterinary Medicine; ${ }^{3}$ Division of Rheumatology and Immunology, Medical University of South Carolina; ${ }^{4}$ Medical University of South Carolina

\subsection{6/lupus-2019-Ism.70}

Background Spontaneous murine models of lupus-like disease are used to study the pathogenesis and genetic causes of SLE. Environmentally-induced lupus models (i.e. pristine-induced) are also valuable to study potential triggers of SLE. Both require extended observation. Some accelerants, (i.e. Type I IFN) are used to trigger earlier disease onset. We used a TLR $7 / 8$ agonist (R848), previously reported to induce lupus-like

Abstract 70 Figure 1 Treatment with Resiquimod (RQ) is associated with a marked proliferation of mononuclear cells consistent with histiocytic sarcoma. In acetone treated mice, the normal lymphoid follicular architecture of the spleen is maintained (top, low mag). In these animals, there are rare, scattered cells within the lymphoid follicles that exhibit strong staining for F4/80, consistent with histiocytic cells (bottom). In animals treated with $R Q$, there is effacement of the normal lymphoid follicles by a proliferation of mononuclear cells. In these mice, $80-90 \%$ of spleen cells exhibit strong punctate to diffuse cytoplasmic staining with F4/80 (bottom) consistent with histiocytic cells. The top images are stained with H\&E. The bottom images are stained with an F4/80 antibody using a red chromogen and DAB counterstain. Treatment of lupus prone mice with R848/ resiquimod (RQ) is associated with a marked proliferation of mononuclear cells in spleen consistent with histiocytic sarcoma. 
disease in WT mice within weeks, as an accelerant in lupus prone NZM2410 mice.

Methods Female and male C57BL/6J mice $(n=29)$ and pre-disease NZM2410 ( $n=31,13-15 \mathrm{wk})$ were treated with topical R848 (100 ug) or acetone vehicle $3 \mathrm{x}$ weekly for 8 weeks. 24 hour urine collection was performed to assess proteinuria by ELISA. Blood was collected to assess autoantibody levels, blood counts and renal function. Spleen and bone marrow (BM) cells were isolated for flow cytometric analysis of immune cell subsets. Spleen and renal tissue were analyzed histologically.

Results Compared to vehicle-treated mice, R848-treated B6 and NZM mice had profoundly enlarged spleens $(\mathrm{p}<0.009$ and $\mathrm{p}<0.0001$, respectively) and survival was significantly reduced $(\mathrm{p}<0.009$ and $\mathrm{p}<0.001)$. Treated B6 mice trended towards a higher ANA $(p=0.059)$, but not antidsDNA, while treated NZM mice had higher levels of ANA $(p=0.07)$ and dsDNA $(p=0.004)$. Serum IFN levels were increased in R848-treated NZM mice, correlating with earlier mortality. Albuminuria and renal pathology in treated NZM mice indicated acceleration of nephritis, but not sufficient to cause death. Consistent with this, there was no significant difference in serum BUN or creatinine between treated and untreated groups. Treated NZM mice had significantly reduced number and percent of $\mathrm{B}$ cells isolated from spleen compared with vehicle (4\% vs. $40 \%)$, as well as $\mathrm{T}$ cells $(8 \%$ vs. $31 \%)$ and pDCs $(0.10 \%$ vs. $0.75 \%)$. CD $11 b+$ cells were significantly expanded (66\% vs. $45 \%)$ in BM from treated NZM mice. IHC revealed a massive expansion of $\mathrm{F} 4 / 80+$ cells in spleens from treated NZM. Pathology of spleens in B6 and NZM mice revealed extramedullary hematopoiesis and changes consistent with histiocytic sarcoma.

Conclusions Topical TLR7/8 agonist treatment induced mild autoimmunity in B6 mice and accelerated autoimmunity in NZM2410 mice. Both had a severe immunophenotype and early death most consistent with malignant histiocytosis. Renal disease was accelerated in NZM2410 mice but was not the cause of death. Care should be taken in using TLR7/8 as a disease accelerant in NZM2410 mice as data suggest that this strain is vulnerable to death hastened by myeloprolferative disease rather than nephritis.

Funding Source(s): NIH NIAMS K08AR068471

\section{PREDICTION OF RESPONSE TO RITUXIMAB IN SLE USING A VALIDATED TWO-SCORE SYSTEM FOR INTERFERON STATUS}

${ }^{1}$ Adewonuola Alase*, 'Zoe Wigston, ${ }^{1}$ Agata Burska, ${ }^{1}$ Elizabeth Hensor, ${ }^{1}$ Antonios Psarras, ${ }^{1}$ Md Yuzaiful Md Yusof, ${ }^{2}$ John Reynolds, ${ }^{3}$ The MASTERPLANS Consortium, ${ }^{1}$ Miriam Wittmann, ${ }^{2}$ lan N Bruce, ${ }^{1}$ Edward Vital. ${ }^{1}$ University of Leeds; ${ }^{2}$ University of Manchester; ${ }^{3} \mathrm{~N} / \mathrm{A}$

\subsection{6/lupus-2019-Ism.71}

Background Rituximab is used for resistant SLE but clinical response varies. Although biomarkers of time to relapse have been validated, there are few biomarkers to predict initial response. Interferon status may predict response to rituximab and anti-TNF in RA. We previously validated two interferonstimulated gene expression scores (IFN-Score-A and IFNScore-B) that improved prediction of clinical outcomes in SLE. IFN-Score-A included most commonly reported ISGs and predicted flares and glucocorticoid requirements. IFNScore-B included ISGs that respond to multiple IFN subtypes and predicted development of SLE in At-Risk individuals. Diagnosis of SLE was associated with both scores, while only IFN-Score-B was elevated in RA. The British Society for Rheumatology Biologics Registry (BILAG-BR) collects data and samples for rituximab-treated patients in the UK. MASTERPLANS is an MRC-funded consortium to identify predictors of response.

Methods This is a preliminary analysis of the first rituximab-treated patients in the BILAG-BR with complete data. Patients were recruited if they were starting a first cycle of rituximab for active SLE (BILAG A or 2xBILAG B) despite previous cyclophosphamide or mycophenolate mofetil. Disease activity was measured using BILAG-2004. Clinical response was defined as improvement by $\geq 1$ grade in active BILAG-2004 systems with no worsening in other systems.

Whole blood was collected into TEMPUS tubes and RNA extracted. IFN-Scores were measured using a custom Taqman array as previously described, normalised to PP1A [El Sherbiny et al. Sci Rep 2018]. Multivariate logistic regression was used to test IFN-Scores and baseline clinical covariates as predictors of BILAG response at 6 months.

Results Samples were available from 147 patients, of whom 84 had complete baseline and 6 month clinical data available

Abstract 71 Table 1 predictors of response

\begin{tabular}{|c|c|c|c|c|c|c|}
\hline Predictor & Non-responders & Responders & Univariable OR $(95 \% \mathrm{Cl})$ & $\mathbf{P}$ & Multivariable OR $(95 \% \mathrm{Cl})$ & $\mathbf{P}$ \\
\hline Age (mean, 95\% Cl) & $40.5(36.2,44.8)$ & $40.9(36.2,45.6)$ & $1.015(0.993,1.037)$ & 0.188 & $0.994(0.959,1.032)$ & 0.765 \\
\hline Baseline organs affected & $22 / 44$ & $23 / 40$ & $0.866(0.468,1.601)$ & 0.645 & $1.024(0.381,2.750)$ & 0.962 \\
\hline \multicolumn{7}{|l|}{ Mucocutaneous } \\
\hline Musculoskeletal & $21 / 44$ & $18 / 40$ & $0.728(0.392,1.354)$ & 0.728 & $0.424(0.145,1.244)$ & 0.118 \\
\hline Renal & $21 / 44$ & $16 / 40$ & $0.869(0.470,1.607)$ & 0.654 & $0.290(0.087,0.969)$ & 0.044 \\
\hline Cardiovascular & $9 / 44$ & $5 / 40$ & $1.250(0.553,2.824)$ & 0.591 & $0.627(0.164,2.391)$ & 0.494 \\
\hline Neurological & $7 / 44$ & $6 / 40$ & $0.859(0.358,2.065)$ & 0.735 & $0.768(0.170,3.473)$ & 0.731 \\
\hline Antimalarial $=Y e s$ & $41 / 44$ & $39 / 40$ & $1.458(0.410,5.186)$ & 0.560 & $13.69(0.995,188.525)$ & 0.050 \\
\hline IFN-Score-A (per unit) & $2.49(1.77,3.19)$ & $1.74(1.10,2.39)$ & $0.845(0.682,1.048)$ & 0.126 & $1.601(0.935,2.743)$ & 0.086 \\
\hline IFN-Score-B (per unit) & $2.36(1.98,2.73)$ & $1.76(1.43,2.09)$ & $0.606(0.394,0.933)$ & 0.023 & $0.267(0.093,0.762)$ & 0.014 \\
\hline
\end{tabular}

